\title{
Unique Luminal Localization of VGAT-C Terminus Allows for Selective Labeling of Active Cortical GABAergic Synapses
}

\author{
Henrik Martens, ${ }^{1}$ Matthew C. Weston, ${ }^{2}$ Jean-Luc Boulland, ${ }^{3}$ Mads Grønborg, ${ }^{4}$ Jens Grosche,,${ }^{5,6}$ Johannes Kacza, ${ }^{7}$ \\ Anke Hoffmann, ${ }^{7}$ Michela Matteoli, ${ }^{8}$ Shigeo Takamori, ${ }^{9}$ Tibor Harkany, ${ }^{10,11}$ Farrukh A. Chaudhry, ${ }^{3}$ \\ Christian Rosenmund, ${ }^{2}$ Christian Erck, ${ }^{1}$ Reinhard Jahn, ${ }^{4}$ and Wolfgang Härtig ${ }^{5}$ \\ ${ }^{1}$ Synaptic Systems GmbH, D-37079 Göttingen, Germany, ${ }^{2}$ Department of Molecular and Human Genetics and Department of Neuroscience, Baylor College \\ of Medicine, Houston, Texas 77030, ${ }^{3}$ Biotechnology Centre of Oslo and Centre for Molecular Biology and Neuroscience, University of Oslo, N-0317 Oslo, \\ Norway, ${ }^{4}$ Department of Neurobiology, Max Planck Institute for Biophysical Chemistry, D-37077 Göttingen, Germany, ${ }^{5}$ Paul Flechsig Institute for Brain \\ Research, University of Leipzig, D-04109 Leipzig, Germany, ${ }^{6}$ Interdisciplinary Center of Clinical Research (IZKF), Faculty of Medicine and ${ }^{7}$ Department of \\ Anatomy, Histology and Embryology, University of Leipzig, D-04103 Leipzig, Germany, ${ }^{8}$ Department of Medical Pharmacology, National Research Council \\ Institute of Neuroscience, University of Milan and Italian Research Hospital Don Gnocchi, I-20129 Milan, Italy, ${ }^{9}$ Center for Brain Integration Research, \\ Graduate School of Medicine, Tokyo Medical and Dental University, Bunkyo-ku, Tokyo 113-8519, Japan, ${ }^{10}$ Department of Medical Biochemistry and \\ Biophysics, Karolinska Institutet, S-17177 Stockholm, Sweden, and ${ }^{1}$ Institute of Medical Sciences, College of Life Sciences and Medicine, University of \\ Aberdeen, Aberdeen AB25 2ZD, United Kingdom
}

Neurotransmitter uptake into synaptic vesicles is mediated by vesicular neurotransmitter transporters. Although these transporters belong to different families, they all are thought to share a common overall topology with an even number of transmembrane domains. Using epitope-specific antibodies and mass spectrometry we show that the vesicular GABA transporter (VGAT) possesses an uneven number of transmembrane domains, with the $\mathrm{N}$ terminus facing the cytoplasm and the $\mathrm{C}$ terminus residing in the synaptic vesicle lumen. Antibodies recognizing the $\mathrm{C}$ terminus of VGAT (anti-VGAT-C) selectively label GABAergic nerve terminals of live cultured hippocampal and striatal neurons as confirmed by immunocytochemistry and patch-clamp electrophysiology. Injection of fluorochromated antiVGAT-C into the hippocampus of mice results in specific labeling of GABAergic synapses in vivo. Overall, our data open the possibility of studying novel GABA release sites, characterizing inhibitory vesicle trafficking, and establishing their contribution to inhibitory neurotransmission at identified GABAergic synapses.

Key words: endocytosis; fluorescence detection; synaptic plasticity; vesicular GABA transporter; live cell imaging; synaptic vesicle

\section{Introduction}

Quantal release of neurotransmitters, concentrated in synaptic vesicles (SVs) (Sudhof, 2004) undergoing activity-dependent exocytosis at synaptic active zones (Schoch and Gundelfinger, 2006), subserves neuronal communication at every synapse. Release of a neurotransmitter from SVs requires either a complete SV fusion and subsequent endocytosis recovering the vesicle membrane (Jahn, 1999), or a "kiss-and-run" mechanism with a transiently open SV fusion pore allowing neurotransmitter release into the synaptic cleft (Klingauf et al., 1998).

Vesicular neurotransmitter transporters (VNTs) shuttle neurotransmitters from the cytosol into SVs (Gasnier, 2000;

Received Aug. 15, 2008; accepted 0ct. 22, 2008.

This work was supported by the Bioprofile Program (BMBF; \#0313132), EU Synapse (LSHM-CT-2005-019055), the Interdisciplinary Center of Clinical Research at the Faculty of Medicine of the Universität Leipzig (210), the Alzheimer's Association, and the Swedish Medical Research Council. We gratefully thank Matthias Böddener and Ute Bauer for technical help and Sonja Wojcik (Max Planck Institute for Experimental Medicine, Göttingen, Germany) for providing VGAT knock-out tissue samples.

This article is freely available online through the J Neurosci Open Choice option.

Correspondence should be addressed to Dr. Henrik Martens, Synaptic Systems GmbH, Rudolf Wissell Strasse 28, D-37079 Göttingen, Germany. E-mail: lab@sysy.com.

D01:10.1523/JNEUROSCI.3887-08.2008

Copyright $\odot 2008$ Society for Neuroscience $\quad 0270-6474 / 08 / 2813125-07 \$ 15.00 / 0$
Chaudhry et al., 2008a). VNTs, including those of glutamate (VGluT1-3) (Takamori, 2006), acetylcholine (VAChT) (Erickson et al., 1996a), monoamines (VMATs) (Erickson et al. 1996b; Liu and Edwards, 1997), and glycine/GABA (VGAT/VIAAT) (Chaudhry et al. 1998) exhibit characteristic substrate specificities and are often used as phenotypic neuronal markers (Takamori et al. 2000). Understanding the correct structure of VNTs is imperative to deduce their functional domains, to define their physicochemical properties, and to identify their domains transiently exposed on the plasmalemmal surface during SV fusion. Our knowledge on the transmembrane topology of VNTs is largely based on computer predictions, which propose cytosolic localization for both the $\mathrm{N}$ and $\mathrm{C}$ termini of all VNTs and an even number (6-12) of transmembrane domains (McIntire et al., 1997; Masson et al., 1999). Experimental data on VGAT partly support the model predictions by showing that GABAergic SVs can be selectively immunoisolated with antibodies raised against its cytosolic N-terminus extremity (Takamori et al., 2000).

To further resolve the transmembrane topology of VGAT we applied limited proteolysis, a method successfully used to map the topologies of other SV proteins (synaptophysin, synaptogyrin, synaptotagmin-1 [Syt1]). Accordingly, luminal epitopes, 

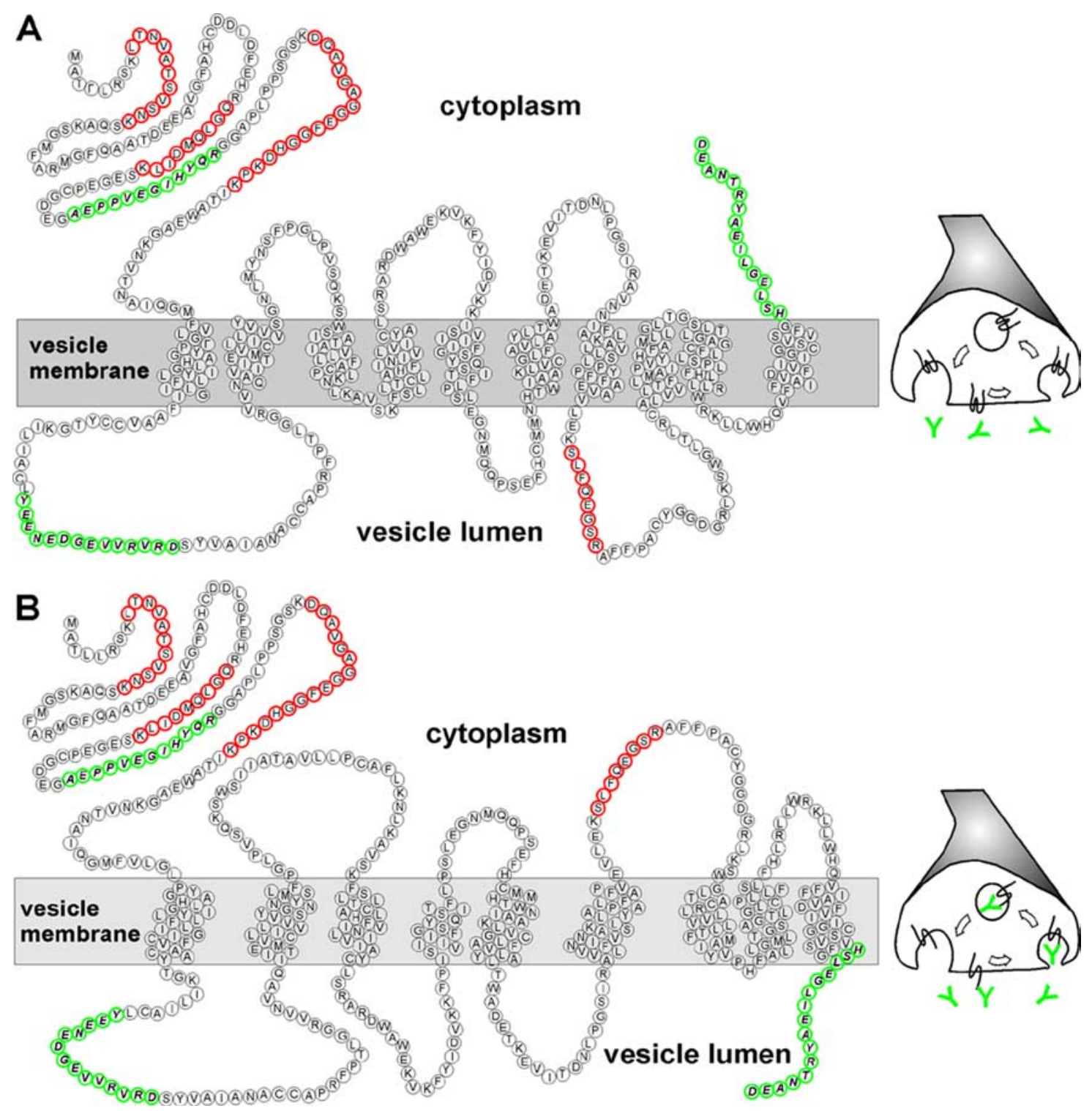

Figure 1. Refined transmembrane topology of VGAT. $\boldsymbol{A}$, Transmembrane structure of VGAT as proposed by Mclntire et al. (1997) and as predicted by the TMHMM algorithm (B). Transmembrane domains are marked in gray. Epitopes in green were used to produce antibodies. Epitopes in red were detected by mass spectrometry. Insets denote the predicted exposure of luminal VGAT epitopes at the plasmalemmal surface and the likelihood of their targeting by antibodies during SV recycling.

e.g., the $\mathrm{N}$ terminus of Syt1 (Perin et al., 1991), are resistant to proteolytic cleavage as revealed by the molecular sizes of protected fragments by Western blotting (Stenius et al., 1995). In contrast, epitopes localized to the outer SV surface facing the cytosol, e.g., the $\mathrm{N}$ - and C-terminal endings of synaptogyrin and synaptophysin, are degradable. Here, we demonstrate that the $\mathrm{C}$ terminus of VGAT folds into the SV lumen. We also developed antibodies against short peptide sequences in (1) the $\mathrm{N}$ terminus, (2) the C terminus, or (3) the first luminal loop of VGAT (VGAT-N, VGAT-C, VGAT-lum1, respectively) (see Fig. 1) to elucidate the membrane topology of this protein, and to visualize forebrain GABAergic synapses both in vitro and in vivo. Similar to antibodies specific for the luminal domain of Syt1, which access the epitope during SV fusion and label endocytic vesicles (Kraszewski et al., 1995), fluorescent anti-VGAT-C targeting an intravesicular epitope allows the selective labeling of inhibitory presynaptic SV clusters during synaptic activity. Patch-clamp electrophysiology supports that anti-VGAT-C selectively recognizes VGAT. Collectively, our data elucidate a refined VGAT transmembrane topology and show that selective targeting of intraluminal VNT epitopes provides novel, effective means to label synapses of living neurons to establish their neurotransmitter phenotype in vivo.

\section{Materials and Methods}

Topology predictions. Transmembrane domain predictions were performed using default settings of the TMHMM (Center for Biological Sequence Analysis, Technical University of Denmark, Lyngby, Denmark; http://www.cbs.dtu.dk/services/TMHMM) and HMMTOP algorithms (Hungarian Academy of Sciences, http://www.enzim.hu/hmmtop/), and the Tmpred service (http://www.ch.embnet.org/software/TMPRED_ form.html). Models were compared with the structure reported by McIntire et al. (1997). Theoretical molecular weights of proteins and proteolytic fragments were calculated with the ProtParam tool (www. expasy.org; Swiss Institute of Bioinformatics, Basel, Switzerland).

Antibodies and fluorochromation. Commercially available antibodies are listed in supplemental material, available at www.jneurosci.org. Peptides corresponding to AA 510-525 (anti-VGAT-C) and AA 171-184 (anti-VGAT-lum1) of rat VGAT (McIntire et al., 1997) were synthesized 
A
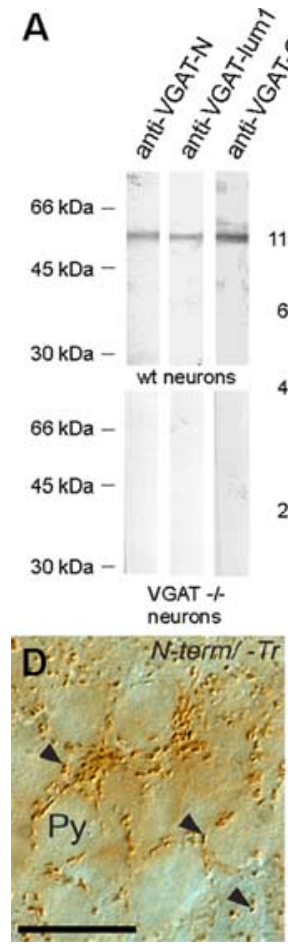

B

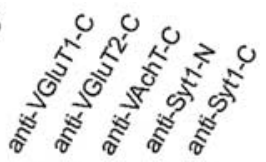

C

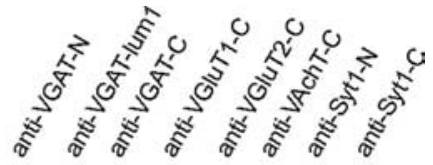

bodies $(5 \mu \mathrm{g} / \mathrm{ml})$ at $37^{\circ} \mathrm{C}$ for $5 \mathrm{~min}$. Cells were repeatedly washed in Krebs-Ringer buffer and subsequently in PBS. Images showing primary fluorochromated antibody binding were captured immediately. Unlabeled primary antibodies were detected after immersion fixation in $4 \%$ paraformaldehyde in PBS, blocking and permeabilization with PBS containing 3\% BSA and $0.1 \%$ Triton $\mathrm{X}-100$ for $20 \mathrm{~min}$, and exposure to Cy3-tagged goat anti-rabbit IgG (1 h, 10-20 $\mu \mathrm{g} / \mathrm{ml}$, Jackson ImmunoResearch). Specific labeling was confirmed by co-staining with monoclonal anti-VGAT-N and Cy5-conjugated goat antimouse IgG. Details of CypHer5E-anti-VGAT-C uptake are in supplemental material, available at www.jneurosci.org.

Electrophysiology. Whole-cell patch-clamp recordings in hippocampal and striatal island cultures prepared from P0 mice were made according to Pyott and Rosenmund (2002) (supplemental material, available at www. jneurosci.org).

In vivo labeling. GABAergic terminals were labeled by stereotaxic injection of antiVGAT-C into the hippocampi of anesthetized (33 $\mu \mathrm{g} / \mathrm{g}$; Hypnomidate, Janssen) young adult C57BL/6N mice. Surgery was performed as described (Siegemund et al., 2006) with $4 \mu \mathrm{g} / 2 \mu \mathrm{l}$ anti-VGAT-C antibody injected at coordinates: $\mathrm{AP}=-2.2 \mathrm{~mm}, \mathrm{~L}=1.9 \mathrm{~mm}, \mathrm{DV}=1.75 \mathrm{~mm}$ (relative to bregma). Mice were transcardially perfused $48 \mathrm{~h}$ later, and tissues processed according to Siegemund et al. (2006). In vivo labeling was analyzed in $30-\mu \mathrm{m}$-thick frozen sections as previously described for cholinergic neurons (Härtig et al., 1998). Labeling specificity was assessed by colocalization of $\mathrm{Cy} 3-$ or Oyster550-anti-VGAT-C $(2 \mu \mathrm{g} / \mathrm{ml})$ and glutamate decarboxylase (GAD; 1:500), VGAT-N (1: $50)$, parvalbumin (1:300) or synaptophysin (1: 500; monoclonal IgGs). The absence of nonspecific in vivo labeling of glutamatergic fibers was shown by using guinea pig-antiand coupled to keyhole limpet hemocyanin using standard procedures (Schneider et al., 1983). Antibodies were custom-generated (Biogenes) and affinity-purified using antigenic peptides immobilized on SulfoLink-Sepharose (Perbio) and are available from Synaptic Systems. Fluorochromation of affinity-purified anti-VGAT-C was performed following the manufacturers protocols (see supplemental material, available at www.jneurosci.org).

Biochemistry. Fractions enriched in synaptic vesicles (LP2) and pure SVs were prepared from adult Wistar rat brains according to Nagy et al. (1976). SDS-PAGE was used to detect full-length proteins (Laemmli, 1970). Pronase-digested SVs were separated by tricine SDS-PAGE to improve resolution in the low molecular range (Schägger and von Jagow, 1987). Each sample contained $200 \mu \mathrm{g}$ of LP2 or $100 \mu \mathrm{g}$ of pronasetreated SVs. Purified SVs (1 mg) were proteolytically digested with 200 $\mu \mathrm{g}$ of pronase (Sigma) in $2.5 \mathrm{ml}$ of PBS ( $0.05 \mathrm{M}, \mathrm{pH} 7.4)$ for $3 \mathrm{~h}$. Reactions were terminated by boiling the samples in SDS sample buffer containing $3 \% \beta$-mercaptoethanol. Western blotting was performed according to Towbin et al. (1979). Mass spectrometry is described in supplemental material, available at www.jneurosci.org.

Immunoperoxidase staining. Immunoperoxidase staining of freefloating Vibratome sections was performed according to Chaudhry et al. (1998) (supplemental material, available at www.jneurosci.org).

Cell culturing and VGAT uptake. Primary hippocampal neurons were cultured according to Gauthier-Campbell et al. (2004). Internalization of fluorochromated antibodies was initiated by incubating neurons in Krebs-Ringer solution containing $55 \mathrm{~mm} \mathrm{KCl}$ (Kraszewski et al., 1995) and polyclonal anti-VGAT-N, anti-VGAT-C or anti-VGAT-lum1 anti-

VGLUT1 (1:500; supplemental Table 1, available at www.jneurosci.org as supplemental material).

\section{Results}

Refined VGAT topology and verification by pronase mapping and mass spectrometry

McIntire et al. (1997) initially suggested a transmembrane VGAT structure with both termini facing the cytoplasm (Fig. $1 A$ ). Based on the TMHMM algorithm, we predicted an alternative transmembrane topology with the $\mathrm{C}$ terminus residing in the SV lumen being transiently exposed on the extracellular plasmalemmal surface during exocytotic fusion of SVs (Fig. $1 B)$. We verified the transmembrane structure of VGAT by protease treatment of SVs followed by immunoblot analysis of the resulting fragments using epitope-mapped VGAT-N, VGAT-C and VGAT-lum1 antibodies (Fig. 1). Luminal, Nand cytoplasmic C-terminal tails of Syt 1 , and the $\mathrm{C}$ termini of VGLUT1, VGLUT2 and VAChT served as controls. Specificity of antibodies directed against three different epitopes of VGAT was confirmed by the findings that they recognized identical bands on cultured wild-type neurons but not on $\mathrm{VGAT}^{-1-}$ material (Fig. 2A). Antibodies against VGLUT1, VGLUT2, VAChT and Syt1 revealed bands at the predicted molecular sizes (Perin et al., 1991, Stenius et al., 1995, Taka- 
mori et al., 2000) in fractions enriched in synaptic vesicles (LP2) (Fig. 2 B). Pronase treatment led to the loss of the VGAT-N epitope (Fig. 2C). In contrast, probing of blots with anti-VGAT-C (AA 510-525) and anti-VGAT-lum 1 (AA171-184) yielded distinct bands showing that the epitopes were protected against proteolysis within the SV lumen (Fig. 2C). Because pronase treatment degrades all regions exposed on the vesicle surface, our refined topology model (Fig. $1 B$ ) predicts peptide fragments of $10.4 \mathrm{kDa}$ and $3.9 \mathrm{kDa}$ for the anti-VGAT-lum 1 and anti-VGAT-C, respectively. Indeed, anti-VGAT-lum 1 detected a proteolytic fragment of the expected size. However, the major protected C-terminal fragment migrated as $\mathrm{a} \sim 12 \mathrm{kDa}$ protein while only a weaker signal of the expected size was detectable. No signals were obtained after pronase treatment with antibodies directed against the $\mathrm{C}$ termini of VGLUT1, VGLUT2 and VAChT, in agreement with their predicted cytoplasmic localization.

To confirm that intravesicular epitopes remained protected during pronase treatment we monitored the cleavage of Syt 1 using antibodies specific for the $\mathrm{N}$-terminal intraluminal and C-terminal cytoplasmic domains, respectively. While the cytoplasmic epitope was lost, a fragment of the expected size was detected by the N-terminal antibody (Fig. 2C). Furthermore, trypsin treatment coupled with mass spectrometry of digested fragments revealed four additional cytosolic peptide fragments (supplemental Fig. 1, available at www.jneurosci.org as supplemental material). Three of these fragments covered the cytosolic $\mathrm{N}$ terminus while one corresponds to the third cytoplasmic loop (Fig. $1 \mathrm{~B}$; supplemental Fig. 1, available at www.jneurosci.org as supplemental material) thus lending further support to our refined model of VGAT topology.

\section{Histochemical localization of VGAT C terminus}

Immunoperoxidase (Fig. 2D-G) and immunofluorescence (supplemental Fig. 2, available at www.jneurosci.org as supplemental material) histochemistry of free-floating vibratome sections processed with or without detergent was performed to confirm the native folding of the two termini of VGAT across SV membranes. Since synapses at the tissue surface are cut open by the vibratome blade antibodies can directly access the surface of SVs (Chaudhry et al., 1998). In the absence of a detergent, anti-VGAT-N reveals punctate terminal-like staining concentrated e.g., in the hippocampal pyramidal cell layer (Fig. $2 D$ ) given its free access to the cytosolic VGAT N terminus (Boulland et al., 2008). In contrast, anti-VGAT-C failed to reveal any punctate staining even at high concentrations that otherwise lead to unspecific nuclear staining (Fig. 2E). Terminal-like labeling with anti-VGAT-C when its penetration is facilitated by Triton X-100 bolsters a luminal localization of this epitope (Fig. $2 F, G$ ). Immunofluorescence labeling and laser-scanning microscopy yielded identical results (supplemental Fig. 2, available at www.jneurosci.org as supplemental material). Overall, these data demonstrate that the $\mathrm{C}$ terminus of VGAT is localized in the lumen of SVs.

\section{Dynamic labeling of GABAergic synapses in vitro}

During recycling of SVs, luminal epitopes of SV proteins are temporarily exposed at the cell surface. Proteins recognizing these epitopes can bind to the surface of intact neurons and may become internalized by endocytosis. Indeed, antibodies directed against the N-terminal domain of Sytl are widely used to selectively label recycling vesicles in nerve terminals (Kraszewski et al., 1995). Therefore, we tested whether anti-VGAT-C can be used to selectively label GABA release sites.

Immunohistochemistry on perfusion-fixed brain sections showed that both unlabeled and Oyster550-labeled antiVGAT-C selectively recognize GABAergic axon terminals (supplemental Fig. 3, available at www.jneurosci.org as supplemental material). Next, we incubated live neurons for 5 min with antiVGAT-C $(5 \mu \mathrm{g} / \mathrm{ml})$ with or without Oyster550 label at $37^{\circ} \mathrm{C}$. Upon $\mathrm{K}^{+}$-induced depolarization, bright immunofluorescence reminiscent of synaptic puncta were observed (Fig. $3 A, B$ ). Selective targeting of unlabeled anti-VGAT-C-derived (Fig. $3 A$ ) or Oyster550-anti-VGAT-C (Fig. 3B) to GABAergic synapses was verified by colocalization with anti-VGAT-N. Negligible or no labeling of inhibitory terminals was obtained by applying either cytoplasmic anti-VGAT-N (Fig. 3C) or anti-VGAT-lum1 (data not shown). Application of a CypHer5E-anti-VGAT-C conjugate that only produces fluorescence signal in endocytic vesicles at acidic $\mathrm{pH}$ also revealed terminal labeling (supplemental Fig. 4, available at www.jneurosci.org as supplemental material). These data reinforce the luminal localization of VGAT C terminus and its accessibility to antibodies in active synapses.

To verify that Oyster550-anti-VGAT-C is selectively taken up by GABAergic synapses, antibody- and mock-treated autaptic cultures 

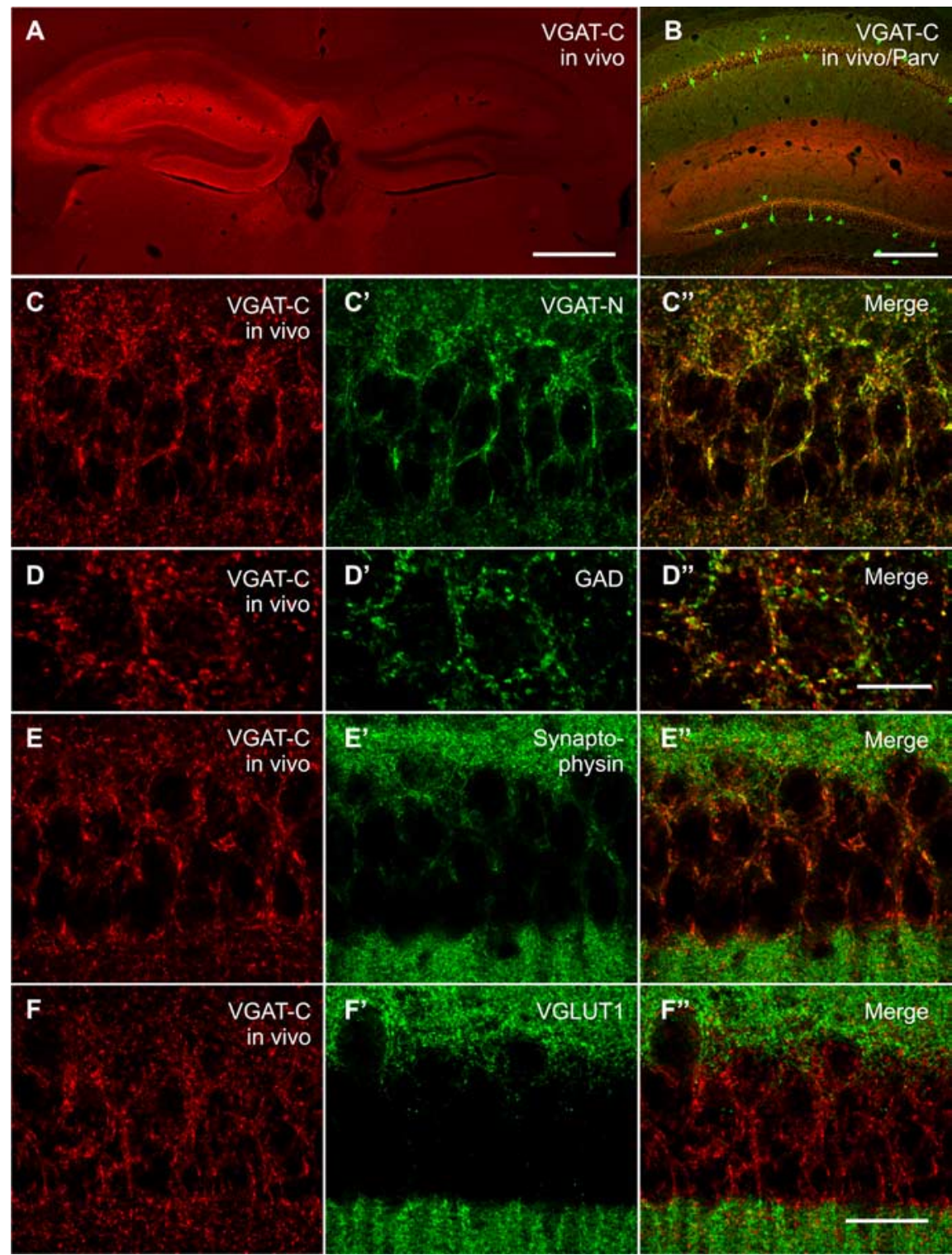

Figure 4. In vivo labeling of GABAergic synapses in mouse hippocampus. Direct in vivo VGAT-C labeling in the CA1 region and dentate gyrus (DG) $48 \mathrm{~h}$ after intrahippocampal antibody infusion combined with indirect Cy2-immunostaining of parvalbumin, VGAT-N, GAD, synaptophysin, and VGLUT1. A, Distribution of Oyster550-anti-VGAT-C in the injected (left) hippocampal hemisphere. $\boldsymbol{B}$, Cy3-anti-VGAT-C in the injected hippocampal hemisphere counterstained with antibodies against parvalbumin. $\mathbf{C}-\boldsymbol{C}^{\prime \prime}$, Oyster550-anti-VGAT-C counterstained with anti-VGAT-N in the CA1 region and combined with anti-GAD in DG $\left(\boldsymbol{D}-\boldsymbol{D}^{\prime \prime}\right)$. Partial coexpression of Oyster550-anti-VGAT-C and synaptophysin in select GABAergic synapses $\left(\boldsymbol{E}-\boldsymbol{E}^{\prime \prime}\right)$. Lack of coexistence of Oyster550anti-VGAT-C and VGLUT1 in the CA1 region $\left(\boldsymbol{F}-\boldsymbol{F}^{\prime \prime}\right)$. Scale bars: $1 \mathrm{~mm}(\boldsymbol{A}), 250 \mu \mathrm{m}(\boldsymbol{B}), 10 \mu \mathrm{m}(\boldsymbol{D}), 30 \mu \mathrm{m}(\boldsymbol{F}$, also valid for $\boldsymbol{C}, \boldsymbol{E})$.

of striatal and hippocampal neurons were analyzed by whole cell recordings in voltage clamp mode. Evoked synaptic responses recorded from labeled striatal neurons were exclusively of IPSC type whereas hippocampal neurons staying unlabeled after antibody treatment always exhibited EPSCs. Miniature IPSC amplitudes, their frequency and the readily releasable pool remained unchanged in Oyster550 anti-VGAT-C labeled cells, whereas the evoked IPSC charge was reduced by $46 \%$ relative to unlabeled neurons (supplemental Fig. 5, available at www.jneurosci.org as supplemental material). Anti-VGAT-C treated neurons showed a slight, but nonsignificant reduction in vesicular release probability.

\section{In vivo labeling of GABAergic synapses}

Our above experiments suggest that anti-VGAT-C may be a versatile tool to visualize GABAergic terminals also in vivo. There- fore, Oyster550-anti-VGAT-C was stereotaxically injected into the CA1 subfield of the hippocampus of adult mice. Bright red immunolabeling reminiscent of the laminar distribution of GABAergic synapses was evident in the injected hemisphere 48 h later (Fig. $4 A, B$ ), with labeled structures exhibiting coincident immunoreactivities for VGAT-N (Fig. 4C), GAD (Fig. 4D) and synaptophysin (Fig. 4E). Highresolution images revealed anti-VGAT-C immunoreactivity surrounding (rather than overlapping) GAD-immunopositive structures (Fig. 4D). In vivo labeling and VGLUT1 immunostaining produced distribution patterns complementary with that of indirect VGLUT1 localization (Fig. $4 F$ ) underscoring the specificity of anti-VGAT-C immunoreactivity. We conclude that fluorochromated antibodies specific for the C-terminal of VGAT can also be used to label GABAergic terminals in live animals.

\section{Discussion}

Here, we show that the $\mathrm{C}$ terminus of VGAT localizes to the SV lumen resulting in an uneven number of transmembrane domains. We also demonstrate that antibodies directed against this domain recognize VGAT in intact neurons and are sequestered by endocytosis without major long-term perturbation of synaptic functions, making these immunoreagents versatile tools to identify release sites of GABA and glycine and to study the kinetics of SV recycling in active synapses.

Our knowledge about the topology of VNTs is largely based on computer predictions that often lack experimental confirmation. The TMHMM algorithm predicted a VGAT transmembrane topology which shows major differences compared with previous models (McIntire et al., 1997). In contrast to the model proposed by McIntire et al. (1997), which agrees with most algorithms predicting transmembrane topologies, the AA 243-263 region is classified by the TMHMM algorithm as part of the first cytoplasmic loop rather than a transmembrane domain. Consequently, VGAT exhibits an uneven number of transmembrane domains whose orientation downstream from serine 242 is inversed with the C-terminal 14-16 residues being localized intraluminally instead of being exposed on the outer SV surface. Accordingly, the $\mathrm{N}$ and C-terminal and first luminal domains recognized by antiVGAT-N, anti-VGAT-C and anti-VGAT-lum1, respectively, were differentially sensitive to pronase treatment of isolated SVs. Our proteolytic assays and mass spectrometry do not provide conclusive support for the exact membrane topology of VGAT. Nevertheless, these results unequivocally confirm that the entire $\mathrm{N}$ terminus and the loop containing AA 408-415 face the cytoplasm while both epitopes recognized by anti-VGAT-C and antiVGAT-lum 1 are localized in the lumen of SVs. 
The major pronase-resistant C-terminal VGAT fragment migrated as $\sim 10-15 \mathrm{kDa}$ protein. This does not correspond to the expected $\sim 3-4 \mathrm{kDa}$ size of the fragment comprising the luminal $\mathrm{C}$ terminus and the last transmembrane domain which was only visible as a weaker signal. Apparently, the small size of the last putative cytoplasmic loop (5-14 AA, depending on the prediction algorithm) renders it partially resistant to proteolytic cleavage, as also reported for synaptogyrin (Stenius et al., 1995). The larger fragment probably represents the $\mathrm{C}$ terminus together with the preceding cytoplasmic and luminal loops and 3 transmembrane domains with a cumulative molecular weight of $10-11 \mathrm{kDa}$. In contrast, the $\mathrm{C}$ termini of VGLUT1, VGLUT2 and VAChT were completely degraded confirming the cytoplasmic localization of these protein epitopes. Thus, VGAT exhibits an exceptional transmembrane topology compared with other known VNTs.

VGAT is the only member of the SLC32 family with no other close mammalian relatives identified so far. Our observations underline the evolutionary distance between VGAT and other known VNTs, which belong to the SLC17 (VGLUT 1-3) or the SLC18 family (VMAT1, 2 and VAChT) (Gasnier, 2004). These latter transporter families are homologous to bacterial toxin/ drug extruders (Chaudhry et al., 2008b) while VGAT is not. VGAT even shows significant structural differences when compared with other functionally related membrane located GABA transporters (GAT-1, 2, 3 and BGT-1; (Alexander et al., 2007). The refined VGAT topology with an uneven number of transmembrane domains suggests a closer relationship between VGAT and amino acid permeases in plants which share sequence homology (Wipf et al., 2002) and a similar transmembrane topology with a cytoplasmic localization of the $\mathrm{N}$ terminus and a $\mathrm{C}$ terminus residing on the outer surface of the plasmalemma (Chang and Bush, 1997). The transmembrane topology for VGAT may also implicate differences in its functional regulation compared with other VNTs whose cytosolic C termini are accessible for other regulatory proteins potentially modulating their function; e.g., endophilin regulation of VGLUT1 recycling (Voglmaier et al., 2006).

Short and transient exposure of live neurons to anti-VGAT-C is sufficient for antibody binding and internalization. Labeling is highly selective for GABAergic neurons indicating that antiVGAT-C endocytosis is mediated by specific epitope binding and not by unspecific fluid phase uptake. Fluorochromated antiVGAT-C exhibited a remarkable penetration in vivo. In a subset of synapses, anti-VGAT-C labeling closely surrounded, though did not entirely overlap, GAD immunoreactivity. This may be due to a sterical competition of internalized and perfusion-fixed anti-VGAT-C and subsequently applied anti-GAD. Interestingly, antibody uptake led to a $46 \%$ reduction of the overall evoked IPSC amplitude and slightly but not significantly reduced SV release probability. The individual miniature fusion events, their frequency and the readily releasable vesicle pool remained unchanged indicating that the presence of luminal anti-VGAT-C can lead to subsequent reduction of the efficiency of SV release but does not affect the translocation of GABA into SVs.

Understanding the precise spatial localization of the VGAT C terminus to the SV lumen is vital for exploiting the potential of our approach to live cell imaging and physiology models of inhibitory neurotransmission. Our confocal imaging studies present the possibility of using VGAT as a novel biomarker in studies aimed at elucidating organizing principles of inhibitory synapses. Combined use of in vivo labeling and tracer ligands holds promise for revealing novel synaptic circuitries and refined innervation patterns in the brain. Moreover, in vivo labeling of functionally intact and electrophysiologically active GABAergic terminals will yield a new dimension of understanding key features of inhibitory neurotransmission, and their perturbations in neuropathological conditions.

\section{References}

Alexander SP, Mathie A, Peters JA (2007) Guide to receptors and channels (GRAC), 2nd edition (2007 Revision). Br J Pharmacol 150:S1-S168.

Boulland J L, Jenstad M, Boekel AJ, Wouterlood FG, Edwards RH, StormMathisen J, Chaudhry FA (2008) Vesicular glutamate and GABA transporters sort to distinct sets of vesicles in a population of presynaptic terminals. Cereb Cortex. Advance online publication. Retrieved May 22, 2008. doi:10.1093/cercor/bhn077

Chang HC, Bush DR (1997) Topology of NAT2, a prototypical example of a new family of amino acid transporters. J Biol Chem 272:30552-30557.

Chaudhry FA, Reimer RJ, Bellocchio EE, Danbolt NC, Osen KK, Edwards RH, Storm-Mathisen J (1998) The vesicular GABA transporter, VGAT, localizes to synaptic vesicles in sets of glycinergic as well as GABAergic neurons. J Neurosci 18:9733-9750.

Chaudhry FA, Boulland JL, Jenstad M, Bredahl MK, Edwards RH (2008a) Pharmacology of neurotransmitter transport into secretory vesicles. Handb Exp Pharmacol 184:77-106.

Chaudhry FA, Edwards RH, Fonnum F (2008b) Vesicular neurotransmitter transporters as targets for endogenous and exogenous toxic substances. Annu Rev Pharmacol Toxicol 48:277-301.

Erickson JD, Weihe E, Schäfer MK, Neale E, Williamson L, Bonner TI, TaoCheng JH, Eiden LE (1996a) The VAChT/ChAT "cholinergic gene locus": new aspects of genetic and vesicular regulation of cholinergic function. Prog Brain Res 109:69-82.

Erickson JD, Schafer MK, Bonner TI, Eiden LE, Weihe E (1996b) Distinct pharmacological properties and distribution in neurons and endocrine cells of two isoforms of the human vesicular monoamine transporter. Proc Natl Acad Sci U S A 93:5166-5171.

Gasnier B (2000) The loading of neurotransmitters into synaptic vesicles. Biochimie 82:327-337.

Gasnier B (2004) The SLC32 transporter, a key protein for the synaptic release of inhibitory amino acids. Pflugers Arch 447:756-759.

Gauthier-Campbell C, Bredt DS, Murphy TH, El-Husseini Ael-D (2004) Regulation of dendritic branching and filopodia formation in hippocampal neurons by specific acylated protein motifs. Mol Biol Cell 15:2205-2217.

Härtig W, Seeger J, Naumann T, Brauer K, Brückner G (1998) Selective in vivo fluorescence labelling of cholinergic neurons containing $\mathrm{p} 75^{\mathrm{NTR}}$ in the rat basal forebrain. Brain Res 808:155-165.

Jahn R (1999) Recycling of synaptic vesicle membrane within nerve terminals. Brain Res Bull 50:313-314.

Klingauf J, Kavalali ET, Tsien RW (1998) Kinetics and regulation of fast endocytosis at hippocampal synapses. Nature 394:581-585.

Kraszewski K, Mundigl O, Daniell L, Verderio C, Matteoli M, De Camilli P (1995) Synaptic vesicle dynamics in living cultured hippocampal neurons visualized with CY3-conjugated antibodies directed against the lumenal domain of synaptotagmin. J Neurosci 15:4328-4342.

Laemmli UK (1970) Cleavage of structural proteins during the assembly of the head of bacteriophage T4. Nature 227:680-685.

Liu Y, Edwards RH (1997) The role of vesicular transport proteins in synaptic transmission and neural degeneration. Annu Rev Neurosci 20:125-156.

Masson J, Sagné C, Hamon M, El Mestikawy S (1999) Neurotransmitter transporters in the central nervous system. Pharmacol Rev 51:439-464.

McIntire SL, Reimer RJ, Schuske K, Edwards RH, Jorgensen EM (1997) Identification and characterization of the vesicular GABA transporter. Nature 389:870-876.

Nagy A, Baker RR, Morris SJ, Whittaker VP (1976) The preparation and characterization of synaptic vesicles of high purity. Brain Res 109:285-309.

Perin MS, Brose N, Jahn R, Südhof TC (1991) Domain structure of synaptotagmin (p65). J Biol Chem 266:623-629.

Pyott SJ, Rosenmund C (2002) The effects of temperature on vesicular supply and release in autaptic cultures of rat and mouse hippocampal neurons. J Physiol 593:523-535.

Schägger H, von Jagow G (1987) Tricine-sodium dodecyl sulfatepolyacrylamide gel electrophoresis for the separation of proteins in the range from 1 to $100 \mathrm{kDa}$. Anal Biochem 166:368-379. 
Schneider WJ, Slaughter CJ, Goldstein JL, Anderson RG, Capra JD, Brown MS (1983) Use of antipeptide antibodies to demonstrate external orientation of the NH2-terminus of the low density lipoprotein receptor in the plasma membrane of fibroblasts. J Cell Biol 97:1635-1640.

Schoch S, Gundelfinger ED (2006) Molecular organization of the presynaptic active zone. Cell Tissue Res 326:379-391.

Siegemund T, Paulke BR, Schmiedel H, Bordag N, Hoffmann A, Harkany T, Tanila H, Kacza J, Härtig W (2006) Thioflavins released from nanoparticles target fibrillar amyloid beta in the hippocampus of APP/PS1 transgenic mice. Int J Dev Neurosci 24:195-201.

Stenius K, Janz R, Südhof TC, Jahn R (1995) Structure of synaptogyrin (p29) defines novel synaptic vesicle protein. J Cell Biol 131:1801-1809.

Sudhof TC (2004) The synaptic vesicle cycle. Annu Rev Neurosci 27:509-547.
Takamori S (2006) VGLUTs: 'exciting' times for glutamatergic research? Neurosci Res 55:343-351.

Takamori S, Riedel D, Jahn R (2000) Immunoisolation of GABA-specific synaptic vesicles defines a functionally distinct subset of synaptic vesicles. J Neurosci 20:4904-4911.

Towbin H, Staehelin T, Gordon J (1979) Electrophoretic transfer of proteins from polyacrylamide gels to nitrocellulose sheets: procedure and some applications. Proc Natl Acad Sci U S A 76:4350-4354.

Voglmaier SM, Kam K, Yang H, Fortin DL, Hua Z, Nicoll RA, Edwards RH (2006) Distinct endocytic pathways control the rate and extent of synaptic vesicle protein recycling. Neuron 51:71-84.

Wipf D, Ludewig U, Tegeder M, Rentsch D, Koch W, Frommer WB (2002) Conservation of amino acid transporters in fungi, plants and animals. Trends Biochem Sci 27:139-147. 